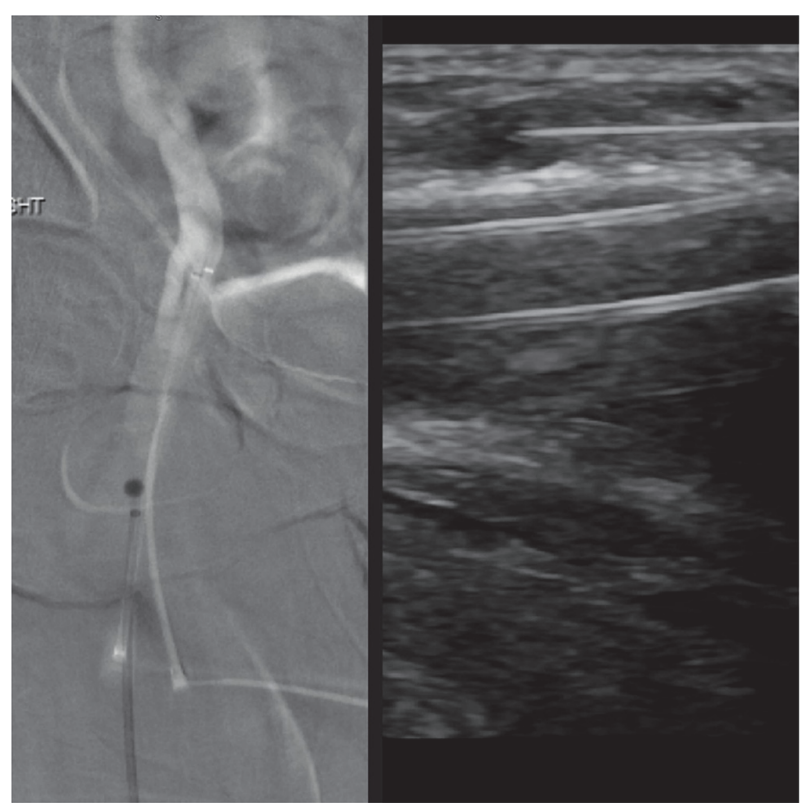

Abstract E-013 Figure 1

were injected with visualization of dynamic increase in artery depth. This allowed for the safe deployment of MynxGrip in Patient C and Angio-Seal VIP in Patient D.

Results The first two cases illustrate how using the roadmap overlay function in fluoroscopy can ensure safe inflation of the intravascular balloon component of the MynxGrip closure device. The last two cases illustrate the use of sonography-guided hydro-dissection to temporarily modify patient anatomy. In all cases, closure devices were safely deployed.

Conclusion Vascular closure device use in patients with unfavorable anatomy is feasible. Safety can be enhanced with image-guided deployment in patients with arterial pathology and in patients with a thin body habitus in some cases traditionally deemed unsafe for closure.

Disclosures L. Lin: None. Z. Wilseck: None. B. Daou: None. A. Pandey: 1; C; NIH, Focused Ultrasound Foundation, American Hydrocephalus Association. J. Gemmete: 1; C; NIH. N. Chaudhary: 1; C; NIH.

\section{E-014 USE OF BASILIC AND CEPHALIC VEIN ACCESS FOR INFERIOR PETROSAL SINUS SAMPLING}

L Lin*, Z Wilseck, J Gemmete, N Chaudhary. Radiology, University of Michigan, Ann Arbor, MI

10.1136/neurintsurg-2021-SNIS.110

Introduction Inferior petrosal sinus (IPS) sampling is performed to confirm the location of excessive ACTH production in the pituitary gland causing Cushing's Syndrome. Traditionally, this procedure is performed via bilateral groin puncture with common femoral vein accesses, which requires a twohour strict bedrest post procedure for recovery. To parallel the evolution to radial access for diagnostic cerebral arterial procedures, venous access in the arm for diagnostic cerebral venous procedures allows for earlier recovery for patients. We

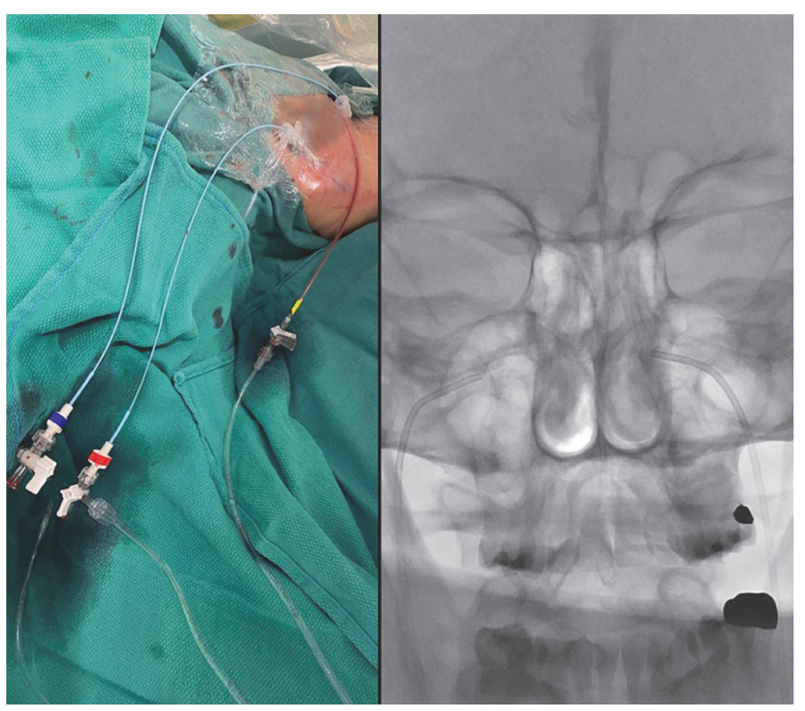

Abstract E-014 Figure 1

describe the technique for basilic and cephalic vein access for IPS sampling.

Materials and Methods Initial sonographic evaluation of potential access sites was performed and planned access sites were marked with skin marker. The arm was then prepped and draped in a sterile fashion. The right basilic and cephalic veins were accessed under sonography with micropuncture kit. A $10 \mathrm{~cm} 5$ French Pinnacle (Terumo Interventional System, Somerset, NJ) sheath was placed in the right basilic vein and $25 \mathrm{~cm} 5$ French Pinnacle sheath was placed in the right cephalic vein. Via the sheaths, under direct fluoroscopy in the AP plane, a 4 French Vertebral catheter was navigated over a 0.035 Glide wire from the cephalic vein to the right internal jugular vein and another 4 French Vertebral catheter was navigated from the basilic vein to the left internal jugular vein. At this point, the lateral fluoroscopy plane was positioned to show the skull base and superior cervical area. The two Vertebral catheters were then navigated under direct fluoroscopic guidance to the respective IPS. Venograms via these catheters were performed to confirm the location. The Vertebral catheters were then secured with adhesive to prevent accidental movement of the catheters from the IPS. Blood samples from both Vertebral catheters as well as peripheral venous sample from the long sheath were obtained simultaneously before and after administration of desmopressin. Post procedure, the catheters and sheaths were removed without complication and hemostasis was obtained with manual compression and placement of wrap dressing.

Results and Conclusion Navigation from the basilic and cephalic veins to the bilateral IPS is possible. This procedure took under 10 minutes of fluoroscopy time with total air kerma of 144.6 mGy. There are three main draining veins in the arm: basilic, cephalic, and brachial. Accessing the superficial basilic and cephalic veins decreases the risk for deep vein thrombus. This access strategy allows for decreased recovery time and earlier mobilization for this patient.

Disclosures L. Lin: None. Z. Wilseck: None. J. Gemmete: 1; C; NIH. N. Chaudhary: 1; C; NIH. 